\title{
Influência do uso do microscópio clínico como auxiliar para realizar a cimentação adesiva de pino de fibra de vidro: Uma análise da resistência de união
}

\author{
Influence of using clinical microscope as auxiliary to perform adhesive cementation of fiberglass \\ posts: A bond strength analysis \\ Influencia del uso del microscopio clínico como auxiliar para realizar la cementación adhesiva del \\ poste de fibra de vidrio: Un análisis de la resistencia de la unión
}

Received: 04/01/2021 | Reviewed: 04/09/2021 |Accept: 04/12/2021 | Published: 04/27/2021

\author{
Roberto O. Barreto \\ ORCID: https://orcid.org/0000-0001-6931-9691 \\ Universidade Brasil, Brasil \\ E-mail: dr.robertoobarreto@gmail.com \\ Ana Grasiela da Silva Limoeiro \\ ORCID: https://orcid.org/0000-0003-4633-720X \\ Faculdade de Ilhéus, Brasil \\ E-mail:grasielalimoeiro@gmail.com \\ Wayne Martins Nascimento \\ ORCID: https://orcid.org/0000-0003-4201-4710 \\ São Leopoldo Mandic, Brasil \\ E-mail: waynemartinsn@gmail.com \\ Vagner Mendes \\ ORCID: https://orcid.org/0000-0002-5140-4465 \\ Universidade Estadual do Sudoeste da Bahia, Brasil \\ E-mail: vagmendes@gmail.com \\ Caio Cesar Souza \\ ORCID: https://orcid.org/0000-0002-6445-5157 \\ ENDOBAHIA Cursos, Brasil \\ E-mail: caioitaobim@ hotmail.com \\ Sergio Paulo Hilgenberg \\ ORCID: https://orcid.org/0000-0003-1976-1013 \\ Centro Universitário União da Vitória, Brasil \\ Prof.sergiopaulo@uniuv.edu.br \\ Angela Toshie Araki \\ ORCID: https://orcid.org/0000-0003-4402-7531 \\ Universidade Cruzeiro do Sul, Brasil \\ E-mail: a araki@uol.com.br \\ Ricardo Scarparo Navarro \\ ORCID: https://orcid.org/0000-0001-6931-9691 \\ Universidade Brasil, Brasil \\ E-mail: ricardo.navarro@universidadebrasil.edu.br
}

\begin{abstract}
Resumo
O objetivo deste estudo foi avaliar a influência da utilização do microscópio clínico e de cimentos resinosos com diferentes estratégias de adesão na resistência de união de pinos de fibra de vidro a dentina nos diferentes terços radiculares. As coroas de sessenta caninos foram seccionadas com disco diamantado, as raízes foram instrumentadas e obturadas (Sistema ProDesign Logic 30.05 + obturação com AH Plus). A desobturação foi realizada com broca correspondente ao diâmetro do pino (DC 2, WhitePost). As raízes foram divididas em seis grupos (n=10): Allcem sem uso do microscópio; Allcem com uso do microscópio; Allcem CORE sem uso do microscópio; Allcem CORE com uso do microscópio; RelyX U200 sem uso do microscópio; RelyX U200 com uso do microscópio. As raízes foram seccionadas transversalmente em fatias dos terços cervical, médio e apical e submetidos ao teste de resistência de união na máquina de testes (Kratos- $0,5 \mathrm{~mm} / \mathrm{min} / 100 \mathrm{Kgf}$ ). Foi realizada análise dos padrões de falha das amostras com estereomicroscópio (40 X). Não houve diferença estatisticamente significativa nos terços cervical independente do tipo de cimento usado ( $p>0,05)$. No terço apical com uso do microscópio, o cimento autoadesivo apresentou valores de adesão superiores $(p<0,05)$. Os cimentos convencionais apresentaram valores semelhantes em todos os terços radiculares $(p>0,05)$ e valores inferiores em comparação ao cimento autoadesivo no terço apical $(p<0,05)$. Houve predomínio do padrão de falha mista nos diferentes grupos e terços. Pode-se concluir que o microscópio clinico, o tipo de cimento resinoso e o terço radicular avaliado influenciaram a resistência de união de pinos de fibra de vidro com a dentina radicular.
\end{abstract}


Palavras- chave: Microscópio clínico; Cimento resinoso; Pinos de fibra de vidro; Teste de push-out.

\begin{abstract}
This study aimed to evaluate the influence of the use of the microscope on the bond strength of fiberglass posts to dentin in the different root thirds. The crowns of sixty canines were sectioned with a diamond disc, and the roots were instrumented and filled (ProDesign Logic System 30.05 + obturation with AH Plus). Desobturation was performed with a drill corresponding to the pin diameter (DC 2, WhitePost). The roots were divided into six groups ( $\mathrm{n}=10)$ : ACsm: Allcem and without a microscope; ACcm: Allcem and microscope; ACCsm: Allcem CORE without a microscope; ACCcm: Allcem CORE with microscope; RUsm: RelyX U200 without a microscope; RUcm: RelyX U200 with a microscope. The roots were cross-sectioned into slices of the cervical, middle and apical thirds and subjected to the bond strength test (Kratos- $0.5 \mathrm{~mm} / \mathrm{min} / 100 \mathrm{Kgf}$ ). A stereomicroscope $(40 \mathrm{x})$ was used to analyze the failure patterns of the samples. There was no statistically significant difference in the cervical thirds regardless of the cement used (p> 0.05). The apical third showed higher adhesion values in the group using a microscope and the self-adhesive cement ( $\mathrm{p}$ $<0.05)$. Conventional cement showed similar values in all root thirds ( $p>0.05)$ and lower values compared to selfadhesive cement in the apical third $(\mathrm{p}<0.05)$. There was a predominance of the mixed failure pattern in the different groups and thirds. It can be concluded that the clinical microscope, the type of resin cement and the evaluated root third influenced the bond strength of fiberglass pins with the root dentin.
\end{abstract}

Keywords: Clinical microscope; Resin cement; Fiberglass posts; Push-out test.

\title{
Resume
}

El objetivo de este estudio fue evaluar la influencia del uso del microscopio en la fuerza de unión de los pines de fibra de vidrio a la dentina en los diferentes tercios radiculares. Se seccionaron las coronas de sesenta caninos con un disco de diamante, se instrumentaron y rellenaron las raíces (ProDesign Logic System 30.05 + obturación con AH Plus). La desobturación se realizó con un taladro correspondiente al diámetro del pin (DC 2, WhitePost). Las raíces se dividieron en seis grupos $(\mathrm{n}=10)$ : ACsm: Allcem y sin microscopio; ACcm: Allcem y microscopio; ACCsm: Allcem CORE sin microscopio; ACCcm: Allcem CORE con microscopio; RUsm: RelyX U200 sin microscopio; RUcm: RelyX U200 con microscopio. Las raíces se seccionaron en cortes de los tercios cervical, medio y apical y se sometieron a la prueba de fuerza de unión en la máquina de prueba (Kratos - 0,5 mm / min / $100 \mathrm{Kgf}$ ). El análisis de los patrones de falla de las muestras se realizó con un microscopio estereoscópico $(40 \mathrm{x})$. No hubo diferencia estadísticamente significativa en los tercios cervicales independientemente del cemento utilizado ( $>>0,05)$. En el tercio apical al microscopio y el cemento autoadhesivo mostró mayores valores de adherencia $(\mathrm{p}<0.05)$. Los cementos convencionales mostraron valores similares en todos los tercios radiculares $(p>0.05)$ y valores menores en comparación con el cemento autoadhesivo en el tercio apical $(\mathrm{p}<0.05)$. Predominó el patrón mixto de fallas en los diferentes grupos y tercios. Se puede concluir que el microscopio clínico, el tipo de cemento resinoso y el tercio radicular evaluado influyeron en la fuerza de unión de los pines de fibra de vidrio con la dentina radicular.

Palabras clave: Microscopio clínico; Cemento resinoso; Clavijas de fibra de vidrio; Prueba de expulsión.

\section{Introdução}

A reabilitação dos dentes tratados endodonticamente representa grande complexidade para clínicos, pois muitas vezes é necessário a utilização de sistemas de retenção do material restaurador coronário à parte radicular como os pinos intrarradiculares (Sicuro et al., 2016; Pereira et al., 2019).

Durante muitos anos, o núcleo metálico fundido tem sido utilizado para reabilitar dentes com comprometimento total ou parcial (Sicuro et al., 2016). Entretanto, algumas desvantagens como a necessidade de maior número de sessões clínicas, custo mais elevado, maior remoção da estrutura dental sadia, maior risco de fratura radicular, devido ao módulo de elasticidade dos retentores metálico e alto índice de falhas catastróficas, além do comprometimento estético tem feito com que a utilização desse sistema fosse limitada (Barjau-Escribano et al., 2006; Ferrari et al, 2012; Sarkis-Onofre et al., 2014; Amarnath et al., 2015).

Dentre os pinos pré-fabricados, os pinos de fibra de vidro são amplamente utilizados para restaurar dentes tratados endodonticamente devido suas vantagens como: propriedades estéticas, resistência à corrosão, fácil remoção, e compatibilidade com sistemas de cimentação adesiva (Malferrari et al. 2003; Lamichhane et al., 2014).

Além disso, o módulo de elasticidade dos pinos de fibra de vidro é semelhante ao da dentina radicular, com melhor distribuição das cargas funcionais para as paredes do canal radicular, em comparação aos retentores intrarradiculares metálicos, 
portanto reduzindo o risco de fraturas graves (Malferrari et al., 2003; Ferrari et al., 2012; Wandscher et al., 2014; Novais et al., 2016).

A capacidade de adesão dos pinos de fibra à dentina é influenciada por diferentes fatores, e os agentes de cimentação são uma parte importante (Skupien et al., 2015; Pereira et al., 2019). Dois grupos de cimentos resinosos são amplamente utilizados: os cimentos resinosos convencionais que envolvem uma técnica com múltiplos passos, sendo, portanto, com técnica mais sensível, e os cimentos autoadesivos que não requerem pré-tratamento dentinário (Barreto et al., 2016; Pereira et al., 2019; Silva et al., (2019)

O fracasso clínico do pino de fibra de vidro esta principalmente relacionado ao seu descolamento (falha na adesão com substrato), que ocorre na interface dentina-cimento e/ou cimento-pino, como resultado da degradação da interface de união resina-dentina, que ocorre ao longo do tempo (Ekambaram et al. 2014; Skupien et al, 2015). Em longo prazo, vários fatores podem comprometer a formação e integridade da interface adesiva, incluindo a má penetração de luz na parte mais profunda da raiz (Pereira et al., 2019), altas tensões de polimerização geradas na interface entre adesivo e cimento (Sicuro et al., 2016), a presença de bolhas e lacunas na camada de cimento (Almeida Junior et al., 2017), a potencial incompatibilidade entre os sistemas adesivos e os cimentos resinosos (Schwartz \& Robbins, 2004), a configuração desfavorável da cavidade (fator C) (Tay et al., 2005) e a dificuldade em controlar a umidade no interior do canal (Pereira et al., 2019).

$\mathrm{Na}$ restauração adesiva do conduto radicular vários fatores podem interferir na resistência de união dos pinos de fibra de vidro cimentado à dentina do canal radicular, como a técnica de instrumentação utilizada e possibilidade de dano térmico a dentina, uso de substâncias químicas irrigadoras e medicações intracanais, a presença da smear layer, resíduos de guta-percha ou cimento obturador, acesso e visualização do operador, materiais adesivos e cimentos utilizados, domínio e treinamento do operador, técnica restauradora. A realização de estudo in vitro avaliando a influência de todos esses fatores podem predizer, indicando ou contra indicando, a utilização de determinada recurso técnico e/ou material. Na avaliação laboratorial por meio de ensaios mecânicos são aplicadas cargas na interface adesiva, gerando estresse entre os materiais e/ou substrato, de forma que quanto maiores os valores de união maior será a resistência dos materiais na interface adesiva frente ao estresse. (Wandscher et al., 2014; Skupien et al, 2015; Barreto et al., 2016; Farid et al., 2018, Silva et al., 2019)

O microscópio clínico é um recurso tecnológico muito utilizado nos procedimentos de endodontia, pois, proporciona maior visibilidade devido a sua capacidade de ampliação e iluminação (Silva et al., 2019), além de melhorar a capacidade do operador de limpar e instrumentar de forma mais eficiente os canais radiculares, e visualizar restos de guta percha em casos de retratamento (Silva et al., 2019).

O presente estudo tem como objetivo avaliar a resistência de união e os padrões de falha do pino de fibra de vidro cimentado com diferentes cimentos resinosos, com o uso do microscópio clínico.

\section{Materiais e Métodos}

\section{Preparo das amostras}

O projeto de pesquisa deste estudo foi aprovado pelo Comitê de Ética e Pesquisa em Seres Humanos da Faculdade São Leopoldo Mandic, recebendo o protocolo de $\mathrm{n}^{\circ}$ : 2.813.342. Sessenta dentes humanos hígidos unirradiculares récem-extraídos foram selecionados e doados pelo Banco de Dentes Humanos da Faculdade de Ilhéus, mantidos em água destilada a $4^{\circ} \mathrm{C}$, trocada semanalmente durante período de tres meses. Foram excluídos do estudo dentes que apresentaram fraturas ou trincas radiculares, tratamento endodôntico prévio, rizogênese incompleta verificadas por microscópio de luz e reabsorções internas ou externas verificadas por radiografia. Os remanescentes de tecido ósseo, gengival e ligamento periodontal foram removidos com curetas periodontais. Os comprimentos radiculares foram padronizados em $17 \mathrm{~mm}$ no sentido ápico-cervical, seccionado as porções 
coronário-radicular excedentes com o auxílio de um paquímetro digital e disco diamantado de dupla face montado em uma peça de mão de baixa velocidade resfriada com um spray de ar/água.

Todos os procedimentos experimentais foram realizados por único operador, treinado e calibrado com a técnica endodôntica e restauradora. Todos os canais radiculares foram instrumentados por um endodontista experiente usando o sistema ProDesign Logic 30.05® (Bassi/Easy Equipamentos, Belo horizonte, Brasil). A exploração inicial dos canais foi feita por um instrumento tipo K \#10 (Dentsply Maillefer, Ballaigues, Suiça), a $2 \mathrm{~mm}$ do comprimento de trabalho (CT), sendo que este foi definido colocando-se o mesmo instrumento no interior do canal até sua ponta aparecer na extremidade, subtraindo-se 1 mm. Esta manobra foi realizada com a utilização do microscópio operatório (Alliance, São Carlos, São Paulo) com magnificação de 8X. A patência do canal foi mantida com o uso do instrumento K \#10 (Dentsply Maillefer, Ballaigues, Suiça), passando 1 mm além do CT. A irrigação foi realizada com o total de $20 \mathrm{~mL}$ de $\mathrm{NaOCl}$ a 2,5\% (Fórmula e Ação®, São Paulo, Brasil). A camada de smear layer foi removida ao final da instrumentação com EDTA a $17 \%$ por 3 minutos, neutralizada ao final com água destilada. As raízes foram secas com papel absorvente e obturados pela técnica do cone único (30.05, Bassi/Easy Equipamentos, Belo Horizonte, Brasil) e cimento AHPlus® (Dentsply Maillefer, Ballaigues, Suiça). O cimento foi colocado no cone e inserido no canal com único movimento. O excesso do cone foi cortado com termo-compactador Touch'n Heat ${ }^{\circledR}$ (SybronEndo, Orange, CA) e as coroas seladas com cimento provisório Coltosol@ (Coltene - Whaledent, Cuyahoga Falls, OH) e armazenados em 100\% de umidade a $37^{\circ}$ por 30 dias. As sessenta raízes foram aleatoriamente distribuídas em seis grupos (n=10) como descrito na Tabela 1:

Tabela 1 - Grupos experimentais.

\begin{tabular}{l|l|l|l}
\hline \multicolumn{2}{l|}{ Grupos } & CIMENTOS RESINOSOS & MICROSCÓPIO CLINICO \\
\hline G1 & AC-sm & ALLCEM (FGM, Joinvile, SC, Brasil) & sem uso do microscópio \\
\hline G2 & AC-cm & ALLCEM (FGM, Joinvile, SC, Brasil)) & com uso do microscópio \\
\hline G3 & ACC-sm & ALLCEM CORE (FGM, Joinvile, SC, Brasil)) & sem uso do microscópio \\
\hline G4 & ACC-cm & ALLCEM CORE (FGM, Joinvile, SC, Brasil)) & com uso do microscópio \\
\hline G5 & RU-sm & RELYX U200 (3M-ESPE) & sem uso do microscópio \\
\hline G6 & RU-cm & RELYX U200 (3M-ESPE) & com uso do microscópio \\
\hline
\end{tabular}

Fonte: Autores.

\section{Procedimentos cimentação dos pinos de fibra de vidro}

Após 7 dias, um único operador, treinado e calibrado com a técnica endodôntica e restauradora realizou a desobturação dos canais radiculares com brocas de largo número 2, 3 e 4, seguida com a broca correspondente ao diâmetro do pino utilizado (DC \#2 WhitePost @- FGM, Joinville, SC, Brasil), a um comprimento de $12 \mathrm{~mm}$, deixando 5 mm de selamento apical. Todos os procedimentos foram realizados pelo mesmo operador.

\section{Todos os grupos}

Brocas de largo (MK Life®) n 6, 5 e 4 foram utilizadas com movimentos circunferenciais na superfície dentinária nas paredes do canal radicular a $5 \mathrm{~mm}, 8 \mathrm{~mm}$ e $12 \mathrm{~mm}$, respectivamente, acionadas por um motor de redução (Endo-motor VDW®, München, Alemanha) a uma velocidade de $1000 \mathrm{rpm}$ e um torque de 2N. Posteriormente, os canais radiculares foram limpos com álcool a 70\% usando um micro aplicador seguido de irrigação abundante com $5 \mathrm{~mL}$ de soro fisiológico 0,9\% por meio do uso de seringas Ultradent ${ }^{\circledR}$ (Ultradent Products Inc., EUA) e agulhas EndoEzeTip® (Ultradent Products, EUA), para remoção dos detritos gerados pelo preparo da superfície dentinária,. Os canais radiculares foram secos com pontas de papel absorvente \# 
80 (Dentsply Maillefer, Ballaigues, Suíça), e os pinos de fibra de vidro foram limpos com álcool à 70\% e em seguida silanizados (Prosil@- FGM, Joinville, SC, Brasil) por um minuto.

Os pinos de fibra de vidro foram cimentados seguindo as orientações dos fabricantes. Nos grupos com o uso do microscópio, a limpeza mecânica foi realizada sob o uso de um microscópio clínico e os grupos sem o uso de microscópio, foram realizados sob iluminação do refletor.

Grupos AC e ACC: (Allcem e Allcem CORE)- cimentos resinoso convencionais que necessitam do condicionamento ácido prévio do substrato dental, realizado com ácido fosfórico ácido fosfórico 37\% (Condac 37®- FGM) por 15s dentro do conduto radicular, em seguida, o conduto foi lavado e removido o excesso de água com cânula aspiradora endodôntica e cones de papel absorvente. Com o aplicador Cavibrush Longo (FGM) foi aplicado o sistema adesivo convencional de frasco único (Ambar®FGM) em toda a extensão do canal radicular condicionado, remoção dos excessos com cone de papel absorvente e foto iniciação na entrada do canal radicular por 40 s com LED Radiiß (SDI, Austrália). Cimento com apresentação em seringa (FGM), seguindo as recomendações do fabricante, manipulando proporções padronizadas do material em bloco de papel, inserindo com broca lentulo o cimento resinoso uniformemente no interior do canal radicular, de apical para cervical, em seguida foi realizada a inserção do pino, com remoção dos excessos e fotoiniciação por 40 s com LED Radiiß.

Grupos RU: (RelyX U200) - cimento resino auto adesivo que dispensa condicionamento com ácido fosfórico prévio do substrato dental e tratamento com sistema adesivo, por conter na sua composição monômeros bifuncionais. O material foi inserido no canal radicular por meio do uso de seringa Centrix ${ }^{\circledR}$ e ponta tipo Accudose®-agulha (Centrix Inc.), com 12 mm de profundidade, até que o refluxo do cimento no terço coronário radicular fosse visto, em seguida, o pino foi inserido, removido os excessos e fotoiniciação por 40 s com LED igual ao grupo anterior. A utilização do equipamento de LED azul foi sempre perpendicular à entrada do canal radicular. Antes da irradiação de cada grupo a intensidade do equipamento foi aferida usando um detector calibrado (MM Optics®; São Carlos, Brasil), e o operador utilizou óculos de proteção apropriado (cor laranja) durante toda a fotoiniciação.

\section{Procedimentos de secção transversal das raízes com pinos de fibra de vidro cimentados}

Após a cimentação, as raízes dos dentes ficaram imersas e armazenados em água destilada a $37^{\circ} \mathrm{C}$ durante 7 dias. A seguir, as amostras foram seccionadas transversalmente através do disco diamantado Lapmaster 4” x 0,012” x 0,5” (102 mm x $0,3 \mathrm{~mm} \times 12,7 \mathrm{~mm}$ ), que foi acoplado à máquina cortadora metalográfica Isomet 1000® (Buehler, Estados Unidos), a fim de se obter uma fatia com espessura de aproximadamente $1 \mathrm{~mm}$ dos terços cervical, médio e apical de cada raiz. As fatias foram individualmente identificadas e armazenadas em água destilada por 7 dias à $37^{\circ} \mathrm{C}$.

\section{Teste de resistência de união push-out}

Passado o período de armazenamento, os espécimes foram submetidos ao teste de resistência adesiva push-out usando uma máquina de Ensaios Universal (Kratos- série KE, Cotia, SP, Brasil) a uma velocidade de 0,5 mm/min e célula de carga de 100 quilogramas força (Kgf). Os corpos de prova foram posicionados em uma base metálica em aço inoxidável contendo um orifício de 2,0 mm de diâmetro interno, sendo o pino posicionado na direção do orifício.

O conjunto foi posicionado na base da máquina de ensaio com carga de $100 \mathrm{Kgf}$. A haste metálica com ponta ativa de $1,0 \mathrm{~mm}$ de diâmetro foi fixada à máquina e posicionada no centro do pino de fibra de vidro. Devido ao formato cônico dos pinos, a carga foi aplicada no sentido do ápice-coroa, a partir da superfície apical, de forma que o pino de fibra de vidro fosse empurrado 
em direção à porção mais larga do conduto. Os valores foram registrados em Kgf e posteriormente convertidos em Megapascal (MPa).

\section{Procedimentos de conversão e análise dos dados}

Os valores obtidos no teste de resistência de união na máquina de ensaios universais foram expressos na unidade quilograma-força (Kgf), foram multiplicados pela constante 9,8 para converter para unidade Newton (N). De acordo com o estudo de Costa et al., (2010), para expressar a tensão de ruptura $(\sigma)$ em MPa, o valor da força máxima para o deslocamento do pino de fibra de vidro em Newtons $(\mathrm{N})$ foi dividido pela área adesiva (A; em $\left.\mathrm{mm}^{2}\right)$ usando a seguinte equação $\sigma=\mathrm{F} / \mathrm{A}$. A fórmula para o cálculo da área foi $\mathrm{A}=\pi(\mathrm{R}+\mathrm{r}) \mathrm{h}$, onde $\pi$ é a constante $3,14, \mathrm{R}$ é o raio do pino cimentado voltado para a região coronal, $\mathrm{r}$ é o raio do pino cimentado para a região apical e h é a espessura da fatia de dentina. O raio maior, menor e a altura da fatia foram mensurados por meio de calibrador digital em milímetros. Os valores obtidos em MPa foram tabelados em planilha de coleta de dados e apresentados de forma descritiva as medidas de tendência central (médias) e de variabilidade (desvio-padrão).

Foi realizada análise do padrão de falha por meio do estereomicroscópio (Carl Zeiss Microscopia Gmbh, Alemanha) com aumento padrão de 40x, analisada por um avaliador calibrado, experiente e cego para os grupos amostrais. Foram realizadas as seguintes análises qualitativa (padrão de falha) e qualitativa (numero de falhas por grupo) para classificação dos padrões de falhas:

I) adesiva entre cimento e dentina;

II) adesiva entre pino e cimento;

III) mista: associação entre fratura adesiva e coesiva;

IV) coesiva: no corpo da dentina;

V) coesiva: no corpo do pino;

VI) coesiva: no corpo do cimento.

Os dados obtidos nos padrões de falha foram tabelados em planilha de coleta de dados e apresentados de forma descritiva a frequência de cada padrão por grupo experimental. Os dados do teste de resistência de união e da análise do padrão de falha foram submetidos a análise estatística inferencial com teste de normalidade e de acordo com sua distribuição na curva foram aplicados os testes estatísticos paramétricos ou não paramétricos utilizando o software IBM SPSS 20.0 (CA, Estados Unidos).

\section{Resultados}

\section{Análise da resistência de união pelo teste de push-out}

Os valores de resistência de união obtidos pelo teste de push-out (MPa) dos diferentes grupos foram analisados estatisticamente (teste de Kolmogorov-Smirnov) e mostraram distribuição normal na curva. A partir disso foram realizados os testes paramétricos de análise de variância ANOVA e teste Tukey ( $\mathrm{p}<0,05)$. Foram realizadas análises por terços (cervical, médio e apical) comparando os diferentes tratamentos (com e sem utilização do microscópio) e os diferentes cimentos resinosos, e por grupos comparando os diferentes terços em cada grupo experimental.

Os resultados encontrados pelo teste de push-out mostram que não houve diferença estatística (p>0,05) entre os grupos nos terços cervical e médio (Gráficos 1 e 2). Houve diferenças significativas entre o grupo AllCem sem microscópio e RelyX com microscópio $(\mathrm{p}<0,01)$ e entre AllCem CORE com microscópio e RelyX com microscópio ( $p<0,05)$ no terço apical (Gráficos 3 e 4). 
Gráfico 1 - Gráfico Box-plot com dados, médias e desvios padrão da resistência de união (MPa) dos diferentes grupos no terço cervical.



AC-sm: Allcem sem microscópio; AC-cm: Allcem com microscópio; ACC-sm: Allcem CORE sem microscópio; ACC-cm: Allcem CORE com microscópio; RU-sm: RelyX U200 sem microscópio; RU-cm: RelyX U200 com microscópio. Não houve diferença estatística significativa entre os grupos ( $p>0,05)$. Fonte: Autores.

Gráfico 2 - Gráfico Box-plot com dados, médias e desvios padrão da resistência de união (MPa) dos diferentes grupos no terço médio.

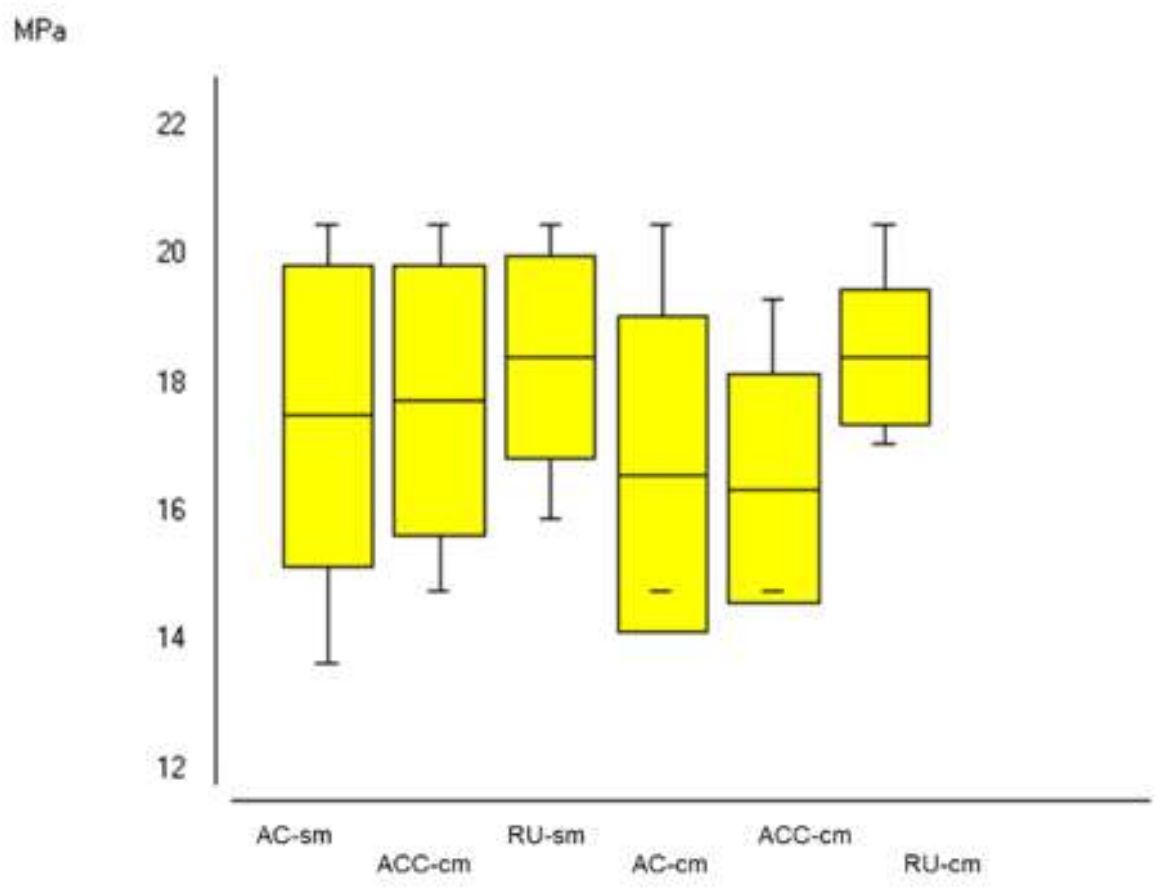

AC-sm: Allcem sem microscópio; AC-cm: Allcem com microscópio; ACC-sm: Allcem CORE sem microscópio; ACC-cm: Allcem CORE com microscópio; RU-sm: RelyX U200 sem microscópio; RU-cm: RelyX U200 com microscópio. Não houve diferença estatística significativa entre os grupos ( $p>0,05)$. Fonte: Autores. 
Gráfico 3 - Gráfico Box-plot com dados, médias e desvios padrão da resistência de união (MPa) dos diferentes grupos no terço apical.

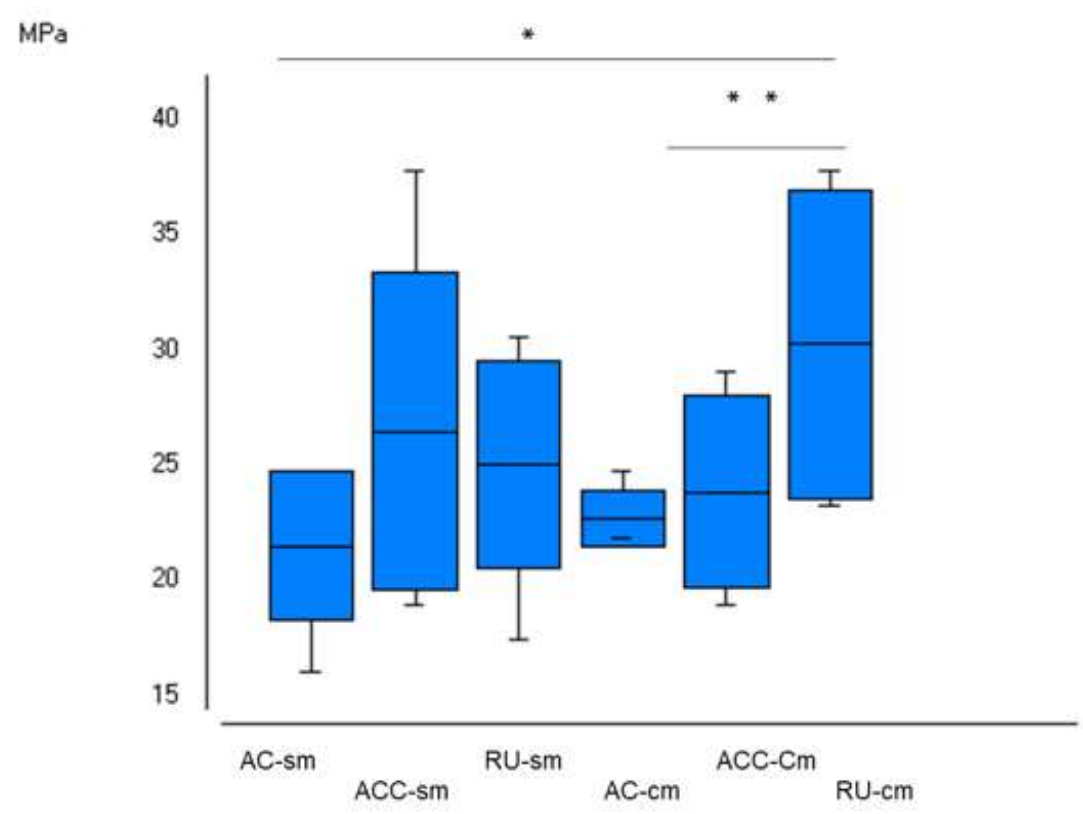

AC-sm Allcem sem microscópio; AC-cm: Allcem com microscópio; ACC-sm: Allcem CORE sem microscópio; ACC-cm: Allcem CORE com microscópio; RU-sm RelyX U200 sem microscópio; RU-cm: RelyX U200 com microscópio. Houve diferenças significativas entre os grupos $*(\mathrm{p}<0,01) * *(\mathrm{p}<0,05)$. Fonte: Autores.

Os valores de resistência de união dos diferentes terços (cervical, médio e apical) foram analisados em cada grupo, sendo observado maiores valores nos terços apicais (Gráfico 4).

Gráfico 4 - Gráfico dos valores de resistência de união (MPa) dos diferentes grupos e terços.

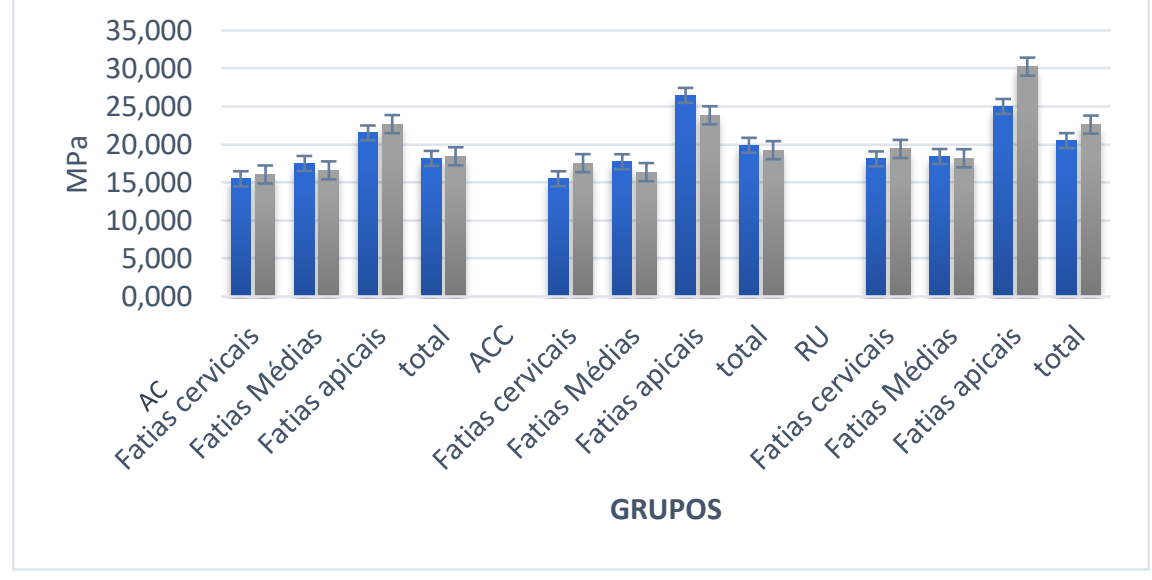

Azul - sem microscópio. Cinza - com microscópio; AC - Allcem; ACC - Allcem CORE; RU - RelyX U200. Fonte: Autores.

Todos os grupos foram submetidos a análise estatística ANOVA e teste Tukey comparando os valores de resistência de união por terços (Gráficos 5 a 10).

Os resultados encontrados pelo teste de push-out mostraram que para todos cimentos e técnicas avaliadas, cimentos convencionais avaliados: AllCem sem microscópio (Gráfico 5), AllCem com microscópio (Gráfico 6), AllCem CORE sem microscópio (Gráfico 7) e AllCem CORE sem microscópio (gráfico 8), e cimentos auto adesivos: RelyX sem microscópio 
(gráfico 9) e RelyX com microscópio (gráfico 10) não houve diferença estatística entre os terços cervical e médio (1 e 2) (p > 0,05), foram observadas diferenças significativas entre os terços cervical e apical (1 e 3) e médio e apical (2 e 3) (p < 0,01), sendo que o terço apical apresentou valores superiores aos demais $(\mathrm{p}<0,01)$.

Gráfico 5 - Análise dos terços do grupo AllCem sem microscópio.

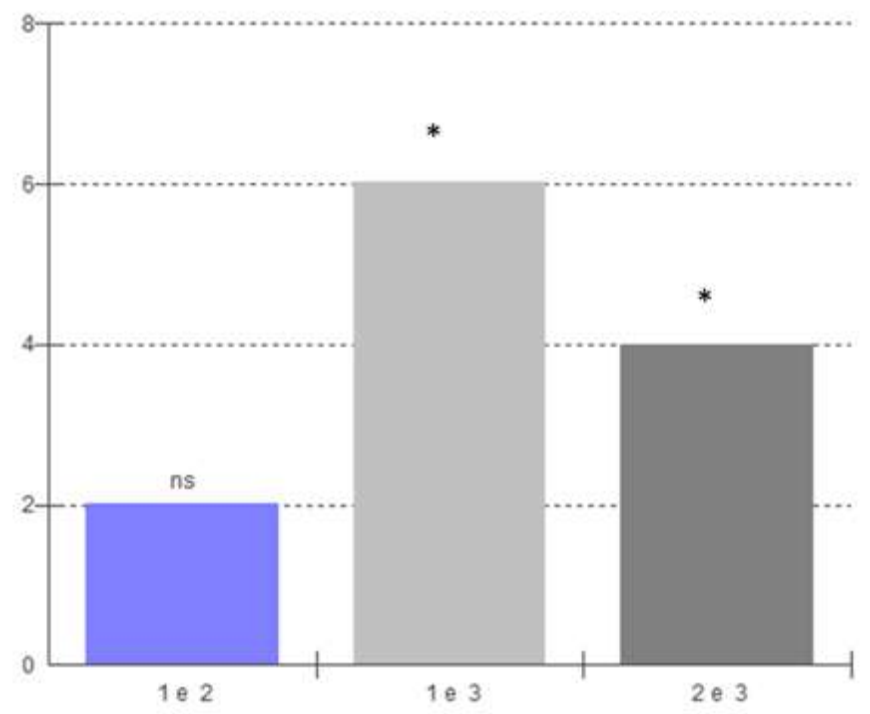

1- Terço cervical; 2 - Terço médio; 3 - Terço apical; (ns) - Não houve diferença estatística entre os terços (p > 0,05); * Houve diferenças significativas entre os terços $(\mathrm{p}<0,01)$. Fonte: Autores.

Gráfico 6 - Análise dos terços do grupo AllCem com microscópio.

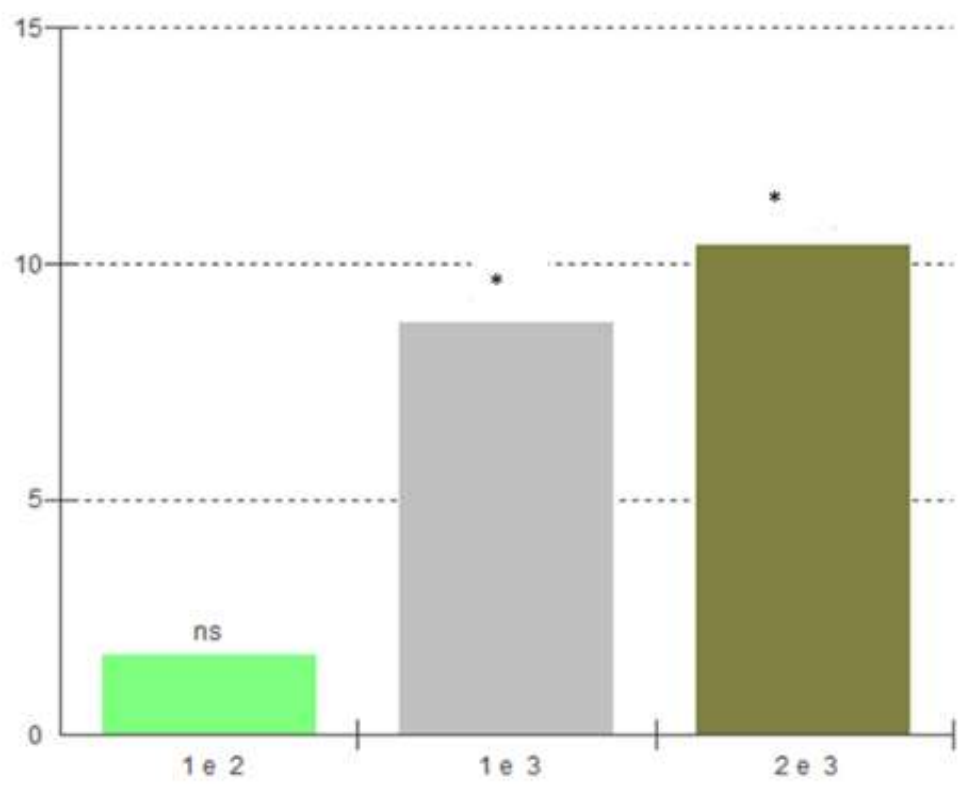

1- Terço cervical; 2 - Terço médio; 3 - Terço apical; (ns) - Não houve diferença estatística entre os terços (p > 0,05); * Houve diferenças significativas entre os terços $(\mathrm{p}<0,01)$, sendo que o terço apical apresentou valores superiores aos demais. Fonte: Autores. 
Research, Society and Development, v. 10, n. 5, e0310514574, 2021

(CC BY 4.0) | ISSN 2525-3409 | DOI: http://dx.doi.org/10.33448/rsd-v10i5.14574

Gráfico 7 - Análise dos terços do grupo AllCem CORE sem microscópio.

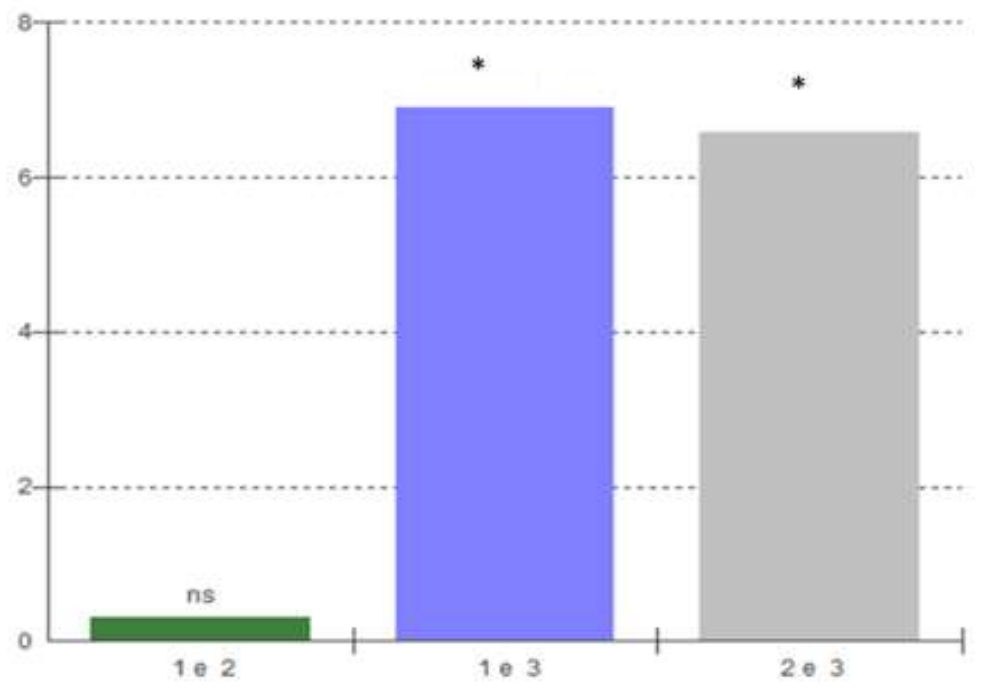

1- Terço cervical; 2 - Terço médio; 3 - Terço apical; (ns) - Não houve diferença estatística entre os terços (p >0,05); * Houve diferenças significativas entre os terços $(\mathrm{p}<0,01)$, sendo que o terço apical apresentou valores superiores aos demais. Fonte: Autores.

Gráfico 8 - Análise dos terços do grupo AllCem CORE com microscópio.

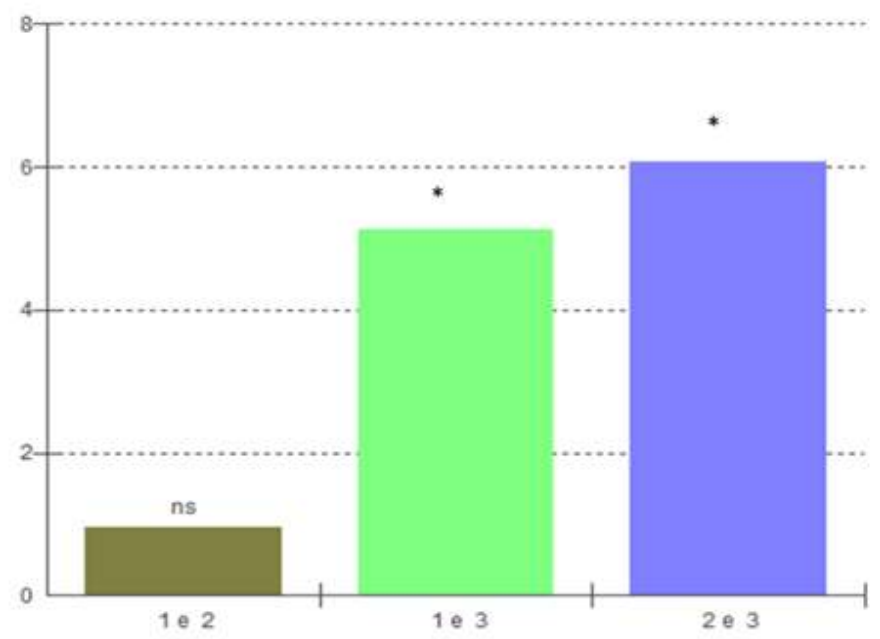

1- Terço cervical; 2 - Terço médio; 3 - Terço apical; (ns) - Não houve diferença estatística entre os terços (p > 0,05); * Houve diferenças significativas entre os terços $(\mathrm{p}<0,01)$. Fonte: Autores. 
Gráfico 9 - Análise dos terços do grupo RellyX sem microscópio.

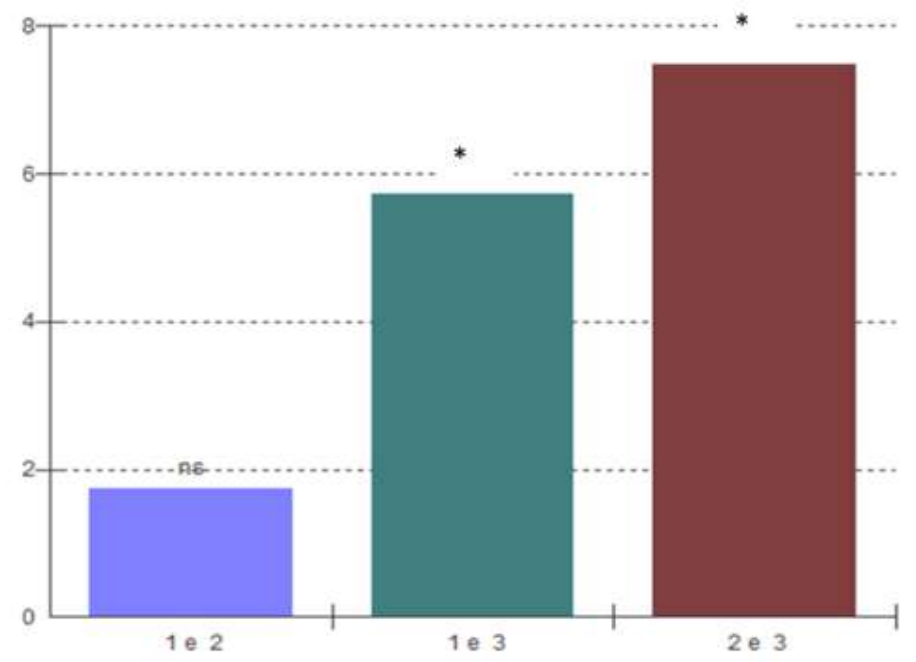

1- Terço cervical; 2 - Terço médio; 3 - Terço apical; (ns) - Não houve diferença estatística entre os terços ( $p>0,05)$; * Houve diferenças significativas entre os terços $(\mathrm{p}<0,01)$, sendo que o terço apical apresentou valores superiores aos demais. Fonte: Autores.

Gráfico 10 - Análise dos terços do grupo RelyX com microscópio.

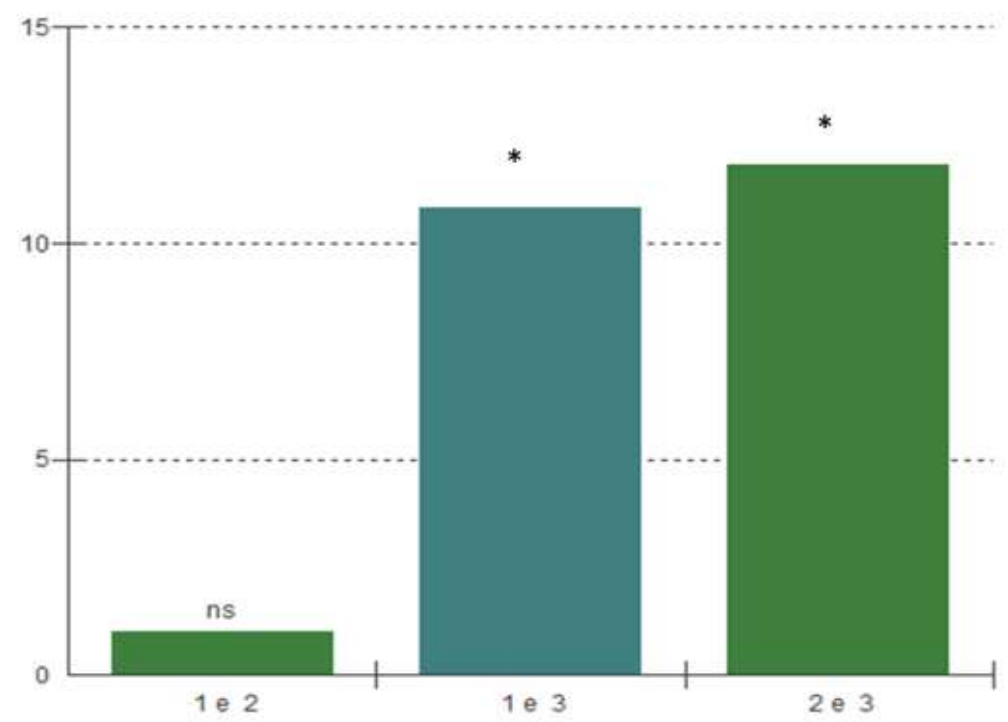

1- Terço cervical; 2 - Terço médio; 3 - Terço apical; (ns) - Não houve diferença estatística entre os terços (p > 0,05); * Houve diferenças significativas entre os terços $(\mathrm{p}<0,01)$. Fonte: Autores.

\section{Análise dos padrões de falha pós teste de resistência de união}

Após o teste de resistência de união push-out as amostras foram avaliadas quanto ao padrão de falha de forma qualitativa e quantitativa. A análise do padrão de falha foi realizada em estereomicroscópio (Carl Zeiss Microscopia Gmbh, Alemanha) com aumento padrão de 40x, por um avaliador calibrado, experiente e cego para os grupos amostrais. As análises qualitativa e qualitativa das interfaces adesivas seguiram a classificação dos padrões de falhas: I) adesiva entre cimento e dentina; II) adesiva entre pino e cimento; III) mista: associação entre fratura adesiva e coesiva; IV) coesiva: no corpo da dentina; V) coesiva: no corpo do pino; VI) coesiva: no corpo do cimento (Figura 1). 
Figura 1 - Imagens representativas dos diferentes padrões de falha no estereomicroscópio (40x).
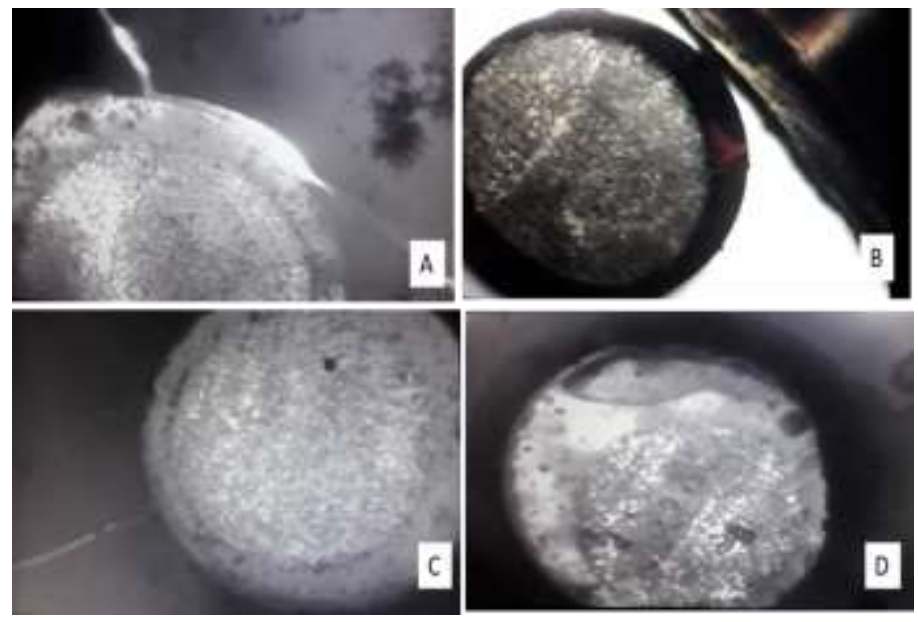

Legenda: A- Mista; B- Adesiva (cimento+dentina); C- Coesiva (corpo da dentina); D- Coesiva (corpo de cimento). Fonte: Autores.

Os dados obtidos nos padrões de falha foram tabelados em planilha de coleta de dados e apresentados de forma descritiva a frequência de cada padrão por grupo experimental. Em todos os grupos experimentais e todos os terços houve predomínio de falhas mistas (Gráfico 11).

Gráfico 11 - Frequências dos padrões de falha nos diferentes grupos experimentais.

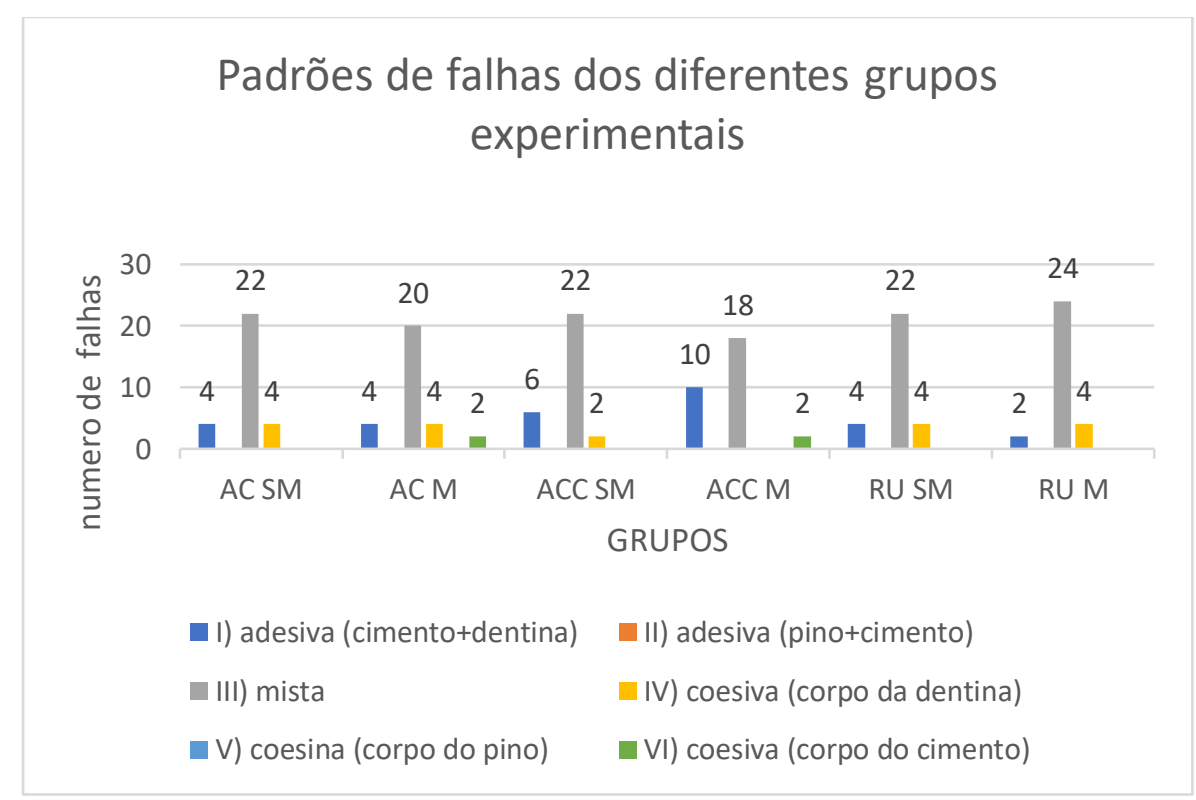

AC SM: Allcem sem microscópio; AC M: Allcem com microscópio; ACC SM: Allcem CORE sem microscópio; ACC M: Allcem CORE com microscópio; RU SM: RelyX U200 sem microscópio; RU M: RelyX U200 com microscópio. Fonte: Autores.

\section{Discussão}

Diante da necessidade do selamento imediato e adequado dos dentes tratados endodonticamente, o uso de pinos de fibra de vidro, imediatamente após o término do tratamento endodôntico, poderá estar indicado a fim de aumentar a previsibilidade do tratamento (Skupien et al, 2015; Marques et al., 2016). O presente estudo objetivou avaliar se a resistência de união, através do teste push-out, seria influenciada pelo uso do microscópio clínico como auxiliar no procedimento de preparo e cimentação de pinos de fibra de vidro no conduto radicular, bem como o desempenho dos cimentos resinosos avaliados. 
O teste de push- out é capaz de distribuir a tensão de forma homogênea, produzindo menor variabilidade nos resultados dos ensaios mecânicos (Goracci et al., 2004). Dessa maneira, o teste de push - out mostra-se o mais adequado para avaliar a resistência adesiva dos pinos de fibra de vidro à dentina radicular (Soares et al., 2008).

O uso do microscópio clínico permite a visualização aprimorada dos sistemas de canais radiculares, o que permite ao operador uma investigação, limpeza, modelação e procedimentos restauradores com maior eficiência (Saunders \& Saunders, 1997).

Ferreira et al. (2015) avaliaram a influência do uso de um microscópio clínico durante a limpeza mecânica das paredes do canal radicular e procedimento de cimentação na resistência de união de pino de fibra de vidro à dentina. Foi observado que com realização da limpeza dos condutos com o microscópio os valores de resistência de união foram significativamente maiores que dos outros grupos sem o uso do microscópio, corroborando com os resultados de resistência de união do presente estudo, sendo que no mesmo estudo de Ferreira et al (2015), o padrão de falha predominante foi adesiva entre cimento e dentina.

Em contrapartida, Silva et al., (2019) compararam a inspeção visual do preparo do conduto para cimentação de pino de fibra de vidro a olho nu; lupa cirúrgica dental com aumento de 3X; microscópio cirúrgico com aumento de $6 \mathrm{X}$. Os resultados mostraram que o método de visualização não teve efeito na resistência de união no teste push-out, e a região cervical apresentou valores superiores em relação a região apical, independentemente do método de inspeção. Tais achados são discordantes do presente estudo que mostrou que com o uso do microscópio clinico durante a execução do preparo radicular promoveu valores superiores de resistência de união dos pinos a dentina no terço apical radicular, ao passo que nos terços cervical e médio radicular não houve influência do uso ou não do microscópio.

Além disso o estudo de Silva, et al., (2019) mostrou predomínio de falha adesiva entre o cimento resinoso auto adesivo RelyX U200 (3M ESPE) e a dentina em todos os grupos, discordante do presente estudo que mostrou predomínio de falhas mistas com o cimento resinoso auto adesivo RelyX U200 (3M ESPE) e os demais cimentos resinosos convencionais testados.

No presente estudo, diferente dos valores de resistência de união, os padrões de fratura não foram influenciados pelo tipo de cimento, portanto, independente do tipo de agente de cimentação utilizado, todos apresentavam reação de presa dual (química e foto iniciação), com diferentes características de pré tratamento do substrato (com condicionamento com ácido fosfórico ou auto condicionante), mecanismos de adesão (micromecânica e/ou química) e propriedades reológicas, como observados nos cimentos utilizados no presente estudo: cimentos resinosos convencionais Allcem (FGM), com maior fluidez, e Allcem CORE (FGM), com maior viscosidade e quantidade de carga, e cimento resinoso auto adesivo RelyX U200 (3M ESPE).

Não foi escopo desta pesquisa avaliar o efeito das soluções irrigadoras, mas outros autores relataram seus efeitos sobre a resistência de união. Marques, et al., (2016) concluíram que o hipoclorito de sódio mostrou um efeito deletério na resistência adesiva de pinos de fibra de vidro cimentados à dentina radicular utilizando os cimentos RelyX U200 e Ambar/Allcem.

Nos terços cervical e médio, não houve diferença estatística em relação a resistência de união do pino de fibra de vidro à dentina para os grupos Allcem, Allcem CORE e RelyX U200, sem e com o uso do microscópio. Esses resultados reafirmaram os de Marques et al., (2016), que compararam o Allcem, Allcem CORE e RelyX U200 e verificaram comportamentos semelhantes em relação a resistência de união entre os cimentos.

Daleprane et al., (2016) compararam o cimento convencional RelyX ARC ao autoadesivo RelyX U200 na cimentação de pinos e também não verificaram diferenças estatísticas nos valores de resistência de união dos cimentos. Esses resultados, contradizem Marques et al., (2016) que compararam os cimentos Âmbar/AllCem e RelyX U200 e verificaram resistência de união superior para o primeiro grupo.

No terço apical, sem o uso do microscópio não houve diferença estatística nos valores de adesão do pino à dentina para os cimentos Allcem, Allcem CORE e RelyX U200. Por sua vez ao utilizar o microscópio clínico foi observada não haver diferença entre os cimentos resinosos convencionais Allcem e Allcem CORE, e esses apresentaram valores de união inferiores 
em comparação ao cimento autoadesivo RelyX U200. Silveira Pedrosa et al., (2016) compararam o uso do cimento RelyX ARC ao cimento autoadesivo RelyX U100, precursor do U200, na cimentação de pinos anatômicos e observaram um desempenho superior para o cimento RelyX U100, como observado no presente estudo no terço apical.

No presente estudo nos terços cervical e médio não foram observadas influencias da técnica, sem ou com uso do microscópio clinico ou tipo do cimento resinoso, nos resultados de resistência de união. Por sua vez, no terço cervical foram observadas influencias da técnica, sendo que o grupo com cimento resinoso auto adesivo (RelyX U200) com uso do microscópio clinico apresentou os maiores valores de resistência de união.

Os resultados de resistência de união encontrados nos grupos com cimentos convencionais duais podem estar relacionada ao fato de que cimentos resinosos apresentarem incompatibilidade de polimerização e união química com os sistemas adesivos simplificados não duais, podendo gerar incompatibilidade química entre os materiais, gerando falhas na interface adesiva. (Van Landuyt et al., 2008; Elsaka 2013; Liu et al., 2014).

No presente estudo, seguindo recomendações do fabricante e técnica de amplo uso clinico, foi realizada a inserção dos cimentos resinosos convencionais com auxílio de broca lentulo, que pode gerar bolhas intrarradiculares e diminuir a resistência de união, especialmente nas porções mais profundas do conduto. Em relação ao cimento auto adesivo foram seguidas orientações do fabricante, com a inserção do cimento resinoso com a ponta aplicadora no interior do conduto, desde as porções mais profundas apicais até cervicais, fato que minimiza a ocorrência de bolhas intrarradiculares. (Malferrari et al., 2003; Ferrari et al., 2012; Wandscher et al., 2014). Assim devem ser encorajados aos clínicos o investimento em cimentos resinosos comerciais duais e que apresentem sistemas de pontas aplicadoras de auto mistura e inserção em porções mais profundas do conduto, devido aos benefícios dos resultados.

Outro fator muito importante são as características e propriedades adesivas do cimentos resinosos testados (Skupien et al, 2015). Os cimentos convencionais duais avaliados no presente necessitam de tratamento prévio com condicionamento ácido e uso de sistema adesivo (etching and rinse), sendo o mecanismo de adesão baseado na formação de camada hibrida pela união micromecâmica ao substrato (Tanoue et al., 2003; Viotti et al., 2009; Ferreira et al. 2012; Marques et al. 2016). O cimentos resinoso autoadesivo utilizado no presente estudo não necessita do pré-tratamento, por apresentar em sua composição sistema adesivo com monômero ácidos e bifuncionais (MDP) que promovem união micromecânica- camada hibrida e união química aos compostos minerais presentes no substrato, trazendo benefícios em relação a técnica e valores de resistência de união. A utilização dos cimentos autoadesivos como RelyX U200 que não requerem pré-tratamento dentinário, pois o sistema adesivo auto-condicionante está incorporado ao cimento, e o processo de ligação na estrutura das fibras de vidro do pino é constituído por uma reação química entre os metacrilatos de fosfato e a hidroxiapatita (De Munck et al, 2004; Viotti et al., 2009; Ferreira et al., 2012; Kadam et al., 2013; Turp et al., 2013; Weiser \& Behr, 2015).

Tais achados corroboram com outros estudos (Gaston et al., 2001; Muniz \& Mathias, 2005; Bitter et al., 2006), que justificam esses maiores valores no terço apical devido ao contato mais próximo entre o pino de fibra e a parede do canal radicular apical, a melhor adaptação do pino e a redução da espessura da camada do cimento são fatores que influenciam no aumento da resistência de união nesse terço. A retenção de fricção parece desempenhar um papel fundamental na retenção total dos pinos de fibra no preparo do canal. Sabendo da relação direta entre atrito e contato superficial, é razoável supor que quanto mais íntimo for o contato entre o cimento resinoso e as paredes do canal, maior será a retenção (Faria-e-Silva et al., 2009). A retenção por atrito pode ser explicada pela absorção de água, que induz a expansão do cimento resinoso (Aleisa et al., 2013). Apesar do uso de brocas fornecidas pelos fabricantes, com tamanho compatível com os pinos de fibra, o que proporciona uma boa adaptação nos terços médio e apical, alguns canais têm grande diâmetro na região cervical. Se o pino não se adapta bem, especialmente no terço cervical, a espessura de cimento fica aumentada e é provável que bolhas estejam presentes, predispondo-o ao descolamento 
(Faria-e-Silva et al., 2009). Quando a camada do agente de cimentação é excessivamente espessa, principalmente na região cervical, o estresse da contração de polimerização é maior (Bouillaguet et al., 2003).

A análise dos padrões de falha mostrou um perfil similar entre os diferentes cimentos, técnica sem ou com uso de microscópio. Em todos os grupos experimentais houve predomínio de falhas do tipo mista nas interfaces adesivas. No terço cervical as falhas foram predominantemente mistas (74\%), seguidas por falhas adesivas em cimento+dentina (23\%) e coesivas no corpo do cimento (3\%), no terço médio as falhas foram do tipo mistas (56\%) seguidas por falhas coesivas em dentina (20\%) e adesivas cimento+dentina (24\%) sendo observado nesse grupo uma menor quantidade de falhas mistas em comparação aos terços cervical e médio; no terço apical as falhas do tipo mista foram as predominantes quase que na totalidade (84\%) seguidas pelas falhas coesiva em dentina (10\%), adesiva cimento+dentina (3\%) e coesiva no cimento (3\%).

Os procedimentos restauradores adesivos com maiores valores de união apresentaram predomínio de falhas do tipo adesivas (sistema adesivo ou cimento resinoso e dentina) ou mistas ou coesivas em dentina. Tais padrões de falhas foram observadas nos diferentes grupos e terços com falhas adesivas nos terços cervical (23\%), médio (24\%) e apical (3\%), as falhas coesivas em dentina foram observadas nos terços médio (20\%) e apical (10\%). Interessante notar o baixo índice de falhas coesivas no material cimento sendo observadas em baixa frequência nos terços cervical (3\%) e apical (3\%) mostrando qualidade nas propriedades dos materiais de cimentação e na sua manipulação e processo de polimerização e presa, no caso do presente estudo todos os cimentos com ativação dual realizada por agentes químicos e por fotoativação com fonte de luz. Não foram observadas falhas coesivas nos pinos e adesivas na interface pino e cemento mostrando uma adequada resistência do pino de fibra de vidro utilizado, e interação do cimento e silano com o substrato vítreo do pino. Sendo que na análise geral do total das amostras mostrou predomínio das fraturas mistas $(n=128)$, seguidas pelas fraturas adesiva (cimento + dentina $)(n=30)$, coesiva (corpo da dentina) $(n=18)$ e coesiva (corpo do cimento) $(n=4)$. Tais achados foram diferentes dos estudos de Ferreira et al., (2015) e Silva et al., (2019), que também avaliaram a influência do uso de um microscópio clínico na limpeza do canal na resistência de união de pino de fibra e mostraram predomínio do padrão de fratura adesivo entre cimento e dentina, enquanto o presente estudo mostrou grande predomínio de fraturas mistas seguido de adesivas entre cimento e dentina. O comportamento superior nos valores de resistência de união à dentina dos cimentos auto-condicionantes no terço apical, considerando a hibridização e união química ao substrato dentinário dos monômeros adesivos, além da maior interação com a superfície dentinária, podem explicar o predomínio na totalidade de falhas mistas para esses grupos.

A presença de fraturas mistas e adesivas (cimento e dentina) mostra adequada interação dos sistemas adesivos e cimentos utilizados com o substrato dentinário e pinos de fibra de vidro, e manutenção da integridade e qualidade adesiva dos materiais e os substratos, com os padrões de falha mista (adesiva e coesiva juntas) e adesiva na interface cimento e dentina as predominantes, bem como a falha coesiva em dentina que mostra adequada hibridização e resistência de união a esse substrato, e ocorrência de fratura no interior do substrato (Pirani et al., 2005).

Ocorreram poucas falhas coesivas no cimento, estas indesejáveis pois demonstram problemas devido falhas na técnica de polimerização ou dificuldade de inserção do cimento (Feilzer et al., 1993) conformação dos canais radiculares (Tay et al., 2005) ou deficiência na intensidade do equipamento de LED (Roberts et al., 2004) gerando fragilidades no corpo do cimento. Como descrito, fatores que podem levar a essas falhas, tais como: a má penetração de luz na parte mais profunda da raiz (Roberts et al., 2004; Faria-e-Silva et al., 2009) altas tensões de polimerização geradas na interface entre adesivo e cimento (Pirani et al. 2005; Feilzer et al., 1993) a presença de bolhas e lacunas na camada de cimento (Patierno et al., 1996).

Embora considerado geralmente de baixa relevância clínica, é claro que resultados obtidos in vitro são úteis para orientar protocolos para várias abordagens clínicas. Então, diante dos resultados deste estudo é recomendado o uso do microscópio clínico no processo de limpeza do conduto radicular e cimentação dos pinos de fibra de vidro utilizando cimento resinoso autoadesivo RelyX U200. 
A reabilitação dos dentes tratados endodonticamente é um procedimento com grande complexidade para os clínicos. A avaliação de técnicas e materiais restauradores, com a introdução de novas tecnologias e equipamentos aplicados à saúde, visam aprimorar os procedimentos clínicos na Odontologia, trazendo resultados com maior qualidade, durabilidade e previsibilidade clínica.

Como no presente estudo in vitro que avaliou o uso do microscópio clinico visando a realização de procedimentos clínicos restauradores buscando maior detalhamento técnico e acuidade visual ao profissional. Como um estudo laboratorial existem limitações e aspectos técnicos que devem ser considerados ao aplicar seus achados na prática clínica. Futuros estudos na linha de reabilitação intraradicular pós tratamento endodôntico devem ser realizados, utilizando o microscópio clinico, e outros recursos de magnificação óptica, com diferentes materiais e protocolos de adesão, tipos de pinos e testes mecânicos para avaliação da eficácia desses recursos, e embasamento para a realização de estudos clínicos randomizados.

\section{Conclusão}

Podemos concluir que a resistência de união do pino de fibra de vidro à dentina radicular foi influenciada pelo uso do microscópio e pelo tipo de cimento resinoso utilizado. O uso do microscópio clinico promoveu valores superiores de adesão no terço apical, não havendo diferença no terço cervical e médio. O cimento autoadesivo apresentou valores de adesão superiores no terço apical, não havendo diferença com os cimentos convencionais no terço cervical e médio.

\section{Referências:}

Amarnath, G. S., Swetha, M. U., Muddugangadhar, B. C., Sonika, R., Garg, A., \& Rao, T. P. (2015). Effect of post material and length on fracture resistance of endodontically treated premolars: an in-vitro study. Journal of international oral health: JIOH, 7(7), 22.

Aktemur, T., Uzunoğlu, E., \& Yılmaz, Z. (2013). Effects of dentin moisture on the push-out bond strength of a fiber post luted with different self-adhesive resin cements. Restorative dentistry \& endodontics, 38(4), 234.

Aleisa, K., Al-Dwairi, Z., Alghabban, R., \& Glickman, G., \& Hsu, M. L. (2013). Effect of cement types and timing of cementation on the retentive bond strength of fiber posts. Journal of Dental Sciences, 7(4), 367-372.

Almeida Junior, L. J. D. S., Penha, K. J. D. S., Souza, A. F., Lula, E. C. O., Magalhães, F. C., Lima, D. M., \& Firoozmand, L. M. (2017). Is there correlation between polymerization shrinkage, gap formation, and void in bulk fill composites? A $\mu \mathrm{CT}$ study. Brazilian oral research, 31.

Barjau-Escribano A., Sancho-Bru J. L., Forner-Navarro L., Rodriguez-Cervantes P. J., Perez-Gonzalez A., \& Sanchez-Marın F.T. (2006) Influence of prefabricated post material on restored teeth: Fracture strength and stress distribution Operative Dentistry 31(1) 47-54.

Barreto M. S., Rosa R. A., Seballos V. G., Machado E., Valandro L. F., Kaizer O. B., Só M. V. R., \& Bier C. A. S. (2016). Effect of Intracanal Irrigants on Bond Strength of Fiber Posts Cemented With a Self-adhesive Resin Cement. Oper Dent 41(6):e159-e167.

Bitter, K., Gläser, C., Neumann, K., Blunck, U., \& Frankenberger, R. (2014). Analysis of resin-dentin interface morphology and bond strength evaluation of core materials for one stage post-endodontic restorations. PLoS One, 9(2), e86294.

Bitter, K., Meyer-Lueckel, H., Priehn, K., Kanjuparambil, J. P., Neumann, K., \& Kielbassa, A. M. (2006). Effects of luting agent and thermocycling on bond strengths to root canal dentine. International endodontic journal, 39(10), 809-818.

Bouillaguet, S., Troesch, S., Wataha, J. C., Krejci, I., Meyer, J. M., \& Pashley, D. H. (2003). Microtensile bond strength between adhesive cements and root canal dentin. Dental Materials, 19(3), 199-205.

Costa, J. A., Rached-Júnior, F. A., Souza-Gabriel, A. E., Silva-Sousa, Y. T. C., \& Sousa-Neto, M. D. (2010). Push-out strength of methacrylate resin-based sealers to root canal walls. International endodontic journal, 43(8), 698-706.

Daleprane, B., Pereira, C. N., Bueno, A. C., Ferreira, R. C., Moreira, A. N., \& Magalhães, C. S. (2016). Bond strength of fiber posts to the root canal: Effects of anatomic root levels and resin cements. The Journal of prosthetic dentistry, 116(3), 416-424.

De Munck, J., Vargas, M., Van Landuyt, K., Hikita, K., Lambrechts, P., \& Van Meerbeek, B. (2004) Bonding of an auto-adhesive luting material to enamel and dentin. Dent Mater. 20(10):963-71.

Ekambaram, M., Yiu, C. K. Y., Matinlinna, J. P., Chang, J. W. W., Tay, F. R., \& King, N. M. (2014). Effect of chlorhexidine and ethanol-wet bonding with a hydrophobic adhesive to intraradicular dentine. Journal of dentistry, 42(7), 872-882.

Elsaka, S.E. (2013) Influence of chemical surface treatments on adhesion of fiber posts to composite resin core materials. Dent Mater. May;29(5):550-8.

Faria-e-Silva, A. L., Pedrosa-Filho, C. D. F., Menezes, M. D. S., Silveira, D. M. D., \& Martins, L. R. M. (2009). Effect of relining on fiber post retention to root canal. Journal of Applied Oral Science, 17(6), 600-604. 
Farid, F., Rostami, K., Habibzadeh, S., \& Kharazifard, M. (2018). Effect of cement type and thickness on push-out bond strength of fiber posts. Journal of dental research, dental clinics, dental prospects, 12(4), 277.

Feilzer, A. J., De Gee, A. J., \& Davidson, C. L. (1993). Setting stresses in composites for two different curing modes. Dental Materials, 9(1), 2-5.

Ferrari, M., Vichi, A., Fadda, G. M., Cagidiaco, M. C., Tay, F. R., Breschi, L., Polimeni, A., \& Goracci, C. (2012) A randomized controlled trial of endodontically treated and restored premolars Journal of Dental Research 91(7) 72-78.

Ferreira, R., Prado, M., de Jesus Soares, A., Zaia, A. A., \& de Souza-Filho, F. J. (2015). Influence of using clinical microscope as auxiliary to perform mechanical cleaning of post space: a bond strength analysis. Journal of endodontics, 41(8), 1311-1316.

Ferreira, R. S., Andreiuolo, R. F., Mota, C. S., Dias, K. R. H. C., Miranda, M. S. (2012) Cimentação adesiva de pinos fibrorreforçados. Rev Bras Odontol. JulDec;69(2):194-8.

Gaston, B. A., West, L. A., Liewehr, F. R., Fernandes, C., \& Pashley, D. H. (2001). Evaluation of regional bond strength of resin cement to endodontic surfaces. Journal of Endodontics, 27(5), 321-324.

Goracci, C., Tavares, A. U., Fabianelli, A., Monticelli, F., Raffaelli, O., Cardoso, P. C., \& Ferrari, M. (2004). The adhesion between fiber posts and root canal walls: comparison between microtensile and push-out bond strength measurements. European journal of oral sciences, 112(4), 353-361.

Kadam, A., Pujar, M., \& Patil, C. (2013). Evaluation of push-out bond strength of two fiber-reinforced composite posts systems using two luting cements in vitro. Journal of conservative dentistry: JCD, 16(5), 444.

Lamichhane, A., Xu, C., \& Zhang, F. Q. (2014). Dental fiber-post resin base material: a review. The Journal of advanced prosthodontics, 6(1), 60.

Liu, C., Liu, H., Qian, Y.T., Zhu, S., \& Zhao, S.Q. (2014) The influence of four dual-cure resin cements and surface treatment selection to bond strength of fiber post. Int J Oral Sci. Mar;6(1):56-60.

Malferrari S., Monaco C., \& Scotti R. (2003) Clinical evaluation of teeth restored with quartz fiber-reinforced epoxy resin posts International Journal of Prosthodontics 16(1) 39-44).

Marques, J. D. N., Gonzalez, C. B., Silva, E. M. D., Pereira, G. D. D. S., Simão, R. A., \& Prado, M. D. (2016). Análise comparativa da resistência de união de um cimento convencional e um cimento autoadesivo após diferentes tratamentos na superfície de pinos de fibra de vidro. Revista de Odontologia da UNESP, 45(2), 121-126.

Muniz, L., \& Mathias, P. (2005). The influence of sodium hypochlorite and root canal sealers on post retention in different dentin regions. Operative dentistryuniversity of washington-, 30(4), 533.

Novais, V. R., Rodrigues, R. B., Simamoto Júnior, P. C., Lourenço, C. S., \& Soares, C. J. (2016). Correlation between the mechanical properties and structural characteristics of different fiber posts systems. Brazilian dental journal, 27(1), 46-51.

Patierno, J. M., Rueggeberg, F. A., Anderson, R. W., Weller, R. N., \& Pashley, D. H. (1996). Push-out strength and SEM evaluation of resin composite bonded to internal cervical dentin. Dental Traumatology, 12(5), 227-236.

Pereira, J. R., Pamato, S., Santini, M. F., Porto, V. C., Ricci, W. A., \& Só, M. V. R. (2019). Push-out bond strength of fiberglass posts cemented with adhesive and self-adhesive resin cements according to the root canal surface. The Saudi Dental Journal.

Pirani, C., Chersoni, S., Foschi, F., Piana, G., Loushine, R. J., Tay, F. R., \& Prati, C. (2005). Does hybridization of intraradicular dentin really improve fiber post retention in endodontically treated teeth?. Journal of Endodontics, 31(12), 891-894.

Roberts, H. W., Leonard, D. L., Vandewalle, K. S., Cohen, M. E., \& Charlton, D. G. (2004). The effect of a translucent post on resin composite depth of cure. Dental Materials, 20(7), 617-622.

Rosenstiel, S. F., Land, M. F., \& Crispin, B. J. (1998). Dental luting agents: A review of the current literature. The Journal of prosthetic dentistry, 80(3), 280301.

Sarkis-Onofre R., Jacinto C., Boscato N., Cenci M. S., \& Pereira-Cenci T. (2014) Cast metal vs glass fibre posts: A randomized controlled trial with up to 3 years of follow up. Journal of Dentistry 42(5) 582-587.

Saunders, W. P., \& Saunders, E. M. (1997). Conventional endodontics and the operating microscope. Dental Clinics of North America, 41(3), 415-428.

Schwartz, R. S., \& Robbins, J. W. (2004). Post placement and restoration of endodontically treated teeth: a literature review. Journal of endodontics, 30(5), 289301.

Sicuro, S. L. M., Gabardo, M. C. L., Gonzaga, C. C., Morais, N. D., Baratto-Filho, F., Nolasco, G. M. C., \& Leonardi, D. P. (2016). Bond strength of selfadhesive resin cement to different root perforation materials. Journal of endodontics, 42(12), 1819-1821.

Silva, L. O., de Souza, B. P., Lima, E. M. C. X., \& de Oliveira, V. M. B. (2013). Protocolos para remoção de retentores intrarradiculares de fibra de vidro: uma revisão crítica. Revista da Faculdade de Odontologia da UFBA, 43(2).

Silva, N. R. D., Rodrigues, M. D. P., Bicalho, A. A., Deus, R. A. D., Soares, P. B. F., \& Soares, C. J. (2019). Effect of Magnification during Post Space Preparation on Root Cleanness and Fiber Post Bond Strength. Brazilian dental journal, 30(5), 491-497.

Silveira-Pedrosa, D. M., Martins, L. R., Sinhoreti, M. A., Correr-Sobrinho, L., Sousa-Neto, M. D., Junior, C. E., \& de Carvalho, J. R. (2016). Push-out Bond Strength of Glass Fiber Posts Cemented in Weakened Roots with Different Luting Agents. The journal of contemporary dental practice.

Skupien, J. A., Sarkis-Onofre, R., Cenci, M. S., \& Moraes, R. R., \& Pereira-Cenci, T. A systematic review of factors associated with the retention of glass fiber posts. Braz Oral Res. 2015;29(1):S1806-83242015000100401. 
Research, Society and Development, v. 10, n. 5, e0310514574, 2021

(CC BY 4.0) | ISSN 2525-3409 | DOI: http://dx.doi.org/10.33448/rsd-v10i5.14574

Soares, C. J., Santana, F. R., Castro, C. G., Santos-Filho, P. C., Soares, P. V., Qian, F., \& Armstrong, S. R. (2008). Finite element analysis and bond strength of a glass post to intraradicular dentin: comparison between microtensile and push-out tests. Dental Materials, 24(10), 1405-1411.

Tanoue, N., Koishi, Y., Atsuta, M., \& Matsumura, H. (2003) Properties of dual-curable luting composites polymerized with single and dual curing modes. $J$ Oral Rehabil. Oct;30(10):1015-21.

Tay, F. R., Loushine, R. J., Lambrechts, P., Weller, R. N., \& Pashley, D. H. (2005). Geometric factors affecting dentin bonding in root canals: a theoretical modeling approach. Journal of endodontics, 31(8), 584-589.

Turp, V., Sen. D., Tuncelli, B., \& Özcan, M. (2013) Adhesion of 10-MDP containing resin cements to dentin .with://dx.doi.org/10.4047/jap.2013.5.3.226

Van Landuyt, K.L., Yoshida, Y., Hirata, I., Snauwaert, J., De Munck, J., Okazaki, M., et al. (2008) Influence of the chemical structure of functional monomers on their adhesive performance. J Dent Res. Aug;87(8):757-61.

Viotti, R. G., Kasaz, A., Pena, C. E., Alexandre, R. S., Arrais, C. A., \& Reis, A. F. (2009) Microtensile bond strength of new self-adhesive luting agents and conventionalmultistep systems. J Prosthet Dent. 102(5):306-12.

Wandscher V. F., Bergoli C. D., Limberger I. F., Ardenghi T. M., \& Valandro L.F. (2014) Preliminary results of the survival and fracture load of roots restored with intracanal posts: Weakened vs nonweakened roots Operative Dentistry 39(5) 541-555.

Weiser, F., \& Behr, M. (2015) Self-adhesive resin cements: a clinical review. J Prosthodont. Feb;24(2):100-8. 